\title{
Pulsations of Proto-Giant-Planets
}

\author{
G. Wuchterl ${ }^{1}$ \\ Institut für Theoretische Astrophysik der Univ. Heidelberg, Germany and \\ Institute for Theoretical Physics, UCSB, Santa Barbara, California
}

\begin{abstract}
Nonlinear oscillations of proto-giant-planets have been found in recent numerical calculations relevant to planetary formation. Pulsations are excited in two phases of the protoplanetary evolution. (a) In an 'instability strip' at core masses of typically $0.2 M_{\oplus}\left(M_{\oplus}\right.$ is the earth mass). Perturbations grow into the nonlinear domain and saturate into perodic variations with relative luminosity-amplitudes of $0.2^{\mathrm{m}}$ (b) At the so called critical mass (typically at $M_{\text {core }} \approx 15 M_{\oplus}$ ). There the pulsations drive a strong mass loss. A large portion of the envelope is ejected. Then the mass loss fades and the envelope settles into a new quasi-equilibrium. This remnant - a post nucleated instability protoplanet - has a compact envelope and is in core and envelope mass similar to Uranus and Neptune.
\end{abstract}

\section{Nonlinear Hydrodynamics of Protoplanetary Pulsations}

Using the methods described be Wuchterl 1991a,b, I followed the evolution of protogiant planets for the 'Jupiter' to 'Neptune' conditions in a standard solar nebula model. The evolutionary sequences where obtained by solving the non-linear, timedependent equations of radiations hydrodynamics in the grey Eddington approximation. Calculations starting at planetesimal-sized cores show static solutions up to core-masses of $0.2 M_{\oplus}$. For the 'Uranus' and 'Neptune' cases pulsations are excited at this core mass as described in the abstract. In the 'Jupiter' and 'Saturn' cases the protoplanetary envelopes remain static until quasi-hydrostatic contraction sets in at core-masses of $\approx 8 M_{\oplus}$.

To study the nature and outcome of the nucleated instability the hydrodynamic evolution starting at the so called critical mass (at $M_{\text {core }} \approx 15 M_{\oplus}$ ) was studied for the same set of solar nebula conditions as above. The onset of mass loss driven by pulsations of the protoplanetary envelope is found in all cases. After a large portion of the envelope mass has been ejected, new more tightly bound envelopes are formed. The excitation mechanism is a $\kappa$-mechanism operating at the Silicate-dust- and $\mathrm{H}^{-}$opacity feature (cf. Wuchterl 1990).

\section{References:}

Wuchterl, G. 1990, Astron. Astrophys., 238, 83-94.

Wuchterl, G. 1991a, Icarus 91, 39-52.

Wuchterl, G. 1991b, Icarus 91, 53-64.

${ }^{1}$ This work was supported by the Deutsche Forschungsgemeinschaft, DFG, project No. Ts 17/2-2 and in part by the National Science Foundation under Grant No. PHY89-04035. 